УДК 616.711-002-036.83:615.825

\section{М.В. Манин ${ }^{1}$, B.В. Абрамов ${ }^{1}$, О.Б. Неханевич ', I.A. Хаитов ${ }^{2}$, Н.И. Каптелов ${ }^{2}$, И.В. Сысенко ${ }^{2}$}

\section{АНАЛИЗ СТРУКТУРЫ ЗАБОЛЕВАЕМОСТИ ДОРСОПАТИЯМИ ПОЯСНИЧНОГО ОТДЕЛА ПОЗВОНОЧНИКА СРЕДИ ПАЦИЕНТОВ ТРУДОСПОСОБНОГО ВОЗРАСТА}

ГУ «Днепропетровская медицинская академия МЗ Украины» ${ }^{1}$

кафедра физической реабилитации, спортивной медицины и валеологии

(зав. - к. мед. н., доч. О.Б. Неханевич)

ул. Дзержинского, 9, Днепропетровск, 49044, Украина

КУ "Днепропетровская областная клиническая больница им. И.И. Мечникова» ${ }^{2}$

пл. Октябрьская, 14, Днепропетровск, 49005, Украина

$S E$ «Dnipropetrovsk medical academy of Health Ministry of Ukraine» ${ }^{1}$

department of physical rehabilitation, sports medicine and valeology

Dzerzhinsky str., 9, Dnipropetrovsk, 49044, Ukraine

CE «Dnepropetrovsk regional clinic named after I.I. Mechnikova» ${ }^{2}$

October's sq. 14, Dnipropetrovsk, 49005, Ukraine

e-mail: olegmed@inbox.ru

Ключевые слова: дорсопатии поясничного отдела позвоночника, реабилитация, лечебная физкультура Key words: lumbar spine dorsopathy, rehabilitation, medical physical culture

Реферат. Аналіз структури захворюваності дорсопатіями поперекового відділу хребта у пацієнтів працездатного віку. Манін М.В., Абрамов В.В., Неханевич О.Б., Хаітов П.О., Каптелов М.І., Сисенко І.В. Метою дослідження був аналіз структури захворюваності дорсопатіями поперекового відділу хребта серед працездатного населення Дніпропетровської області для обтрунтування диферениійованого підходу до вибору методики ЛФК. У статті проаналізована структура захворюваності на дорсопатії у 371 пацієнта в період 2009-2013 рр. У роботі представлений аналіз найбільш частих морфологічних змін у структурах хребта, їх локалізачія та особливості клінічного перебігу. Виявлений зв'язок клінічних проявів дорсопатій зі структурними змінами хребта, стажем захворювання, визначена ефективність реабілітації засобами лікувальної фізкультури залежно від якісних та кількісних ознак захворювання. Результати дослідження вказують, що понад 40\% пацієнтів знаходились на повторному лікуванні, клінічна маніфестація у 62\% хворих спостерігалась у найбільи прачездатному вічі (від 30 до 60 років), відповідно до стажу захворювання збільшувалась кількість загострень на рік, у 57,5\% призначення ЛФК обмежувалось у зв'язку з розвитком супутньої патології, у 40\% випадків на фоні позитивної динаміки больового синдрому відмічались недостатні зміни в об'ємі рухів хребта протягом лікування. Це свідчить про необхідність розробки алгоритму диференційованого підходу до вибору засобів ЛФК при лікуванні дорсопатій поперекового відділу хребта.

Abstract. Analysis of structure of lumbar spine dorsopathy morbidity in able-bodied age patients. Manin M.V., Abramov V.V., Nekhanevich O.B., Khaitov P.A., Kaptelov N.I., Sysenko I.V. The analysis of structure of lumbar spine dorsopathy morbidity among able-bodied population of the Dnepropetrovsk area for substantiation of differentiated approach to the choice of exercise therapy methods was a research objective. The structure of dorsopathy morbidity in 371 patients in 2009-2013 period is analysed in the work. The paper presents analysis of the most frequent morphological changes in the structures of the spine, their localization and clinical course. Link of clinical manifestations of dorsopathy with structural changes of the backbone, the disease experience is revealed, efficiency of rehabilitation by means of physiotherapy exercises depending on qualitative and quantitative signs of disease is defined. Results of the research specify that more than $40 \%$ of patients were on repeated treatment, clinical manifestation in $62 \%$ of patients was observed at the most able-bodied age (from 30 to 50 years), according to the disease experience number of aggravations per a year increased, in 57,5\% of cases administration of medical physical culture was limited due to development of an accompanying pathology, in 40\% of cases against positive dynamics of painful syndrome insufficient changes in volume of movements of the spine segments throughout treatment were marked. It testifies to necessity of working out algorithm of differentiated approach to the choice of means medical physical culture in treatment of lumbar spine dorsopathy.

Актуальность выбранной тематики продиктована тесной связью патологии позвоночника с показателями качества жизни, работоспособности и инвалидизации населения. Многочисленные 
эпидемиологические исследования показывают, что эпизоды неспецифической мышечноскелетной боли в течение жизни беспокоят 60$85 \%$ населения $[1,2,4,5]$. Причинами стойкой утраты работоспособности у лиц с заболеваниями периферической нервной системы в $80 \%$ случаев являются вертеброгенные поражения $[9,10,11]$. Болевые синдромы в нижней части спины (более $70 \%$ случаев дорсалгии) связаны с дегенеративно-дистрофическими изменениями позвоночника и в общей структуре заболеваемости с временной утратой трудоспособности занимают в Украине второе место (уступая только респираторным инфекциям), что имеет огромное клиническое, социальное и экономическое значение для общества [6]. Выраженные клинические проявления наблюдаются преимущественно в период активной трудовой деятельности (возраст 20-50 лет) и представляют собой одну из самых частых причин временной нетрудоспособности, а также выхода на инвалидность, при этом экономические затраты занимают первое место среди затрат на лечение среди всех других заболеваний нервной системы $[1,3]$. Многочисленные данные статистики свидетельствуют об отсутствии тенденции к уменьшению заболеваемости на протяжении последних лет [2, 7, 12, 13]. Высокая инвалидизация лиц трудоспособного возраста вследствие поражений опорно-двигательной системы побудила экспертов ВОЗ объявить 20002010 гг. декадой костно-суставных болезней (The Bone and Joint Decade, Geneva, 2000-2010) [14].

B различных литературных источниках приводятся разноречивые данные относительно взаимосвязи выраженности клинических проявлений с морфологическими изменениями в структурах позвоночника, возрастом, стажем заболевания, отдаленных результатов хирургического лечения, что дает повод к дальнейшему изучению структуры заболеваемости дорсопатиями.

Данная работа выполнена в соответствии с планом научно-исследовательской темы «Медико-биологическое обеспечение физической реабилитации, спортивных и оздоровительных тренировок» (№ 0113U007653) кафедры физической реабилитации, спортивной медицины и валеологии ГУ «Днепропетровская медицинская академия МЗ Украины».

Цель работы - проанализировать структуру заболеваемости дорсопатиями поясничного отдела позвоночника среди трудоспособного населения Днепропетровской области для обоснования дифференцированного подхода к выбору методики ЛФК.

\section{МАТЕРИАЛЫ И МЕТОДЫ ИССЛЕДОВАНИЙ}

Нами были отобраны методом случайной выборки и проанализированы данные 371 пациента, которые находились на лечении в отделении лечебной физкультуры КУ «Днепропетровская областная больница им. И.И. Мечникова» г. Днепропетровска с диагнозом дорсопатия за период с 2010 г. по 2013 г. Методом выкопировки из историй болезни мы получили данные о структуре заболеваемости дорсопатиями, возрасте, поле пациентов, длительности заболевания, характере и локализации морфологических изменений, функциональных нарушениях и синдромах заболевания: болевом, мышечно-тоническом (MT), статико-динамических нарушений (СДН) и их динамики в процессе лечения. Проанализированы данные об эффективности реабилитации при ограничении назначения ЛФК в связи с наличием сопутствующих заболеваний. Статистическую обработку полученных результатов проводили при помощи пакета лицензионных программ STATISTIKA (6.1, серийный номер AGAR909E415822FA). Анализировали вид распределения показателей при помощи Wкритерия Шапиро-Уилка. Определяли достоверности различий между показателями с учетом типа распределения при помощи Т-критерия Стьюдента, U-критерия Манна-Уитни и критерия распределения (хи-квадрат Пирсона). Пороговым уровнем статистической значимости брали $\mathrm{p}<0,05$ [8].

Работа проводилась с учетом нормативных документов комиссии по медицинской этике, разработанной с учетом положений конвенции Совета Европы «О защите прав человека в аспекте биомедицины» (1997 г.) и Хельсинской декларации Всемирной медицинской организации (2008 г.).

\section{РЕЗУЛЬТАТЫ И ИХ ОБСУЖДЕНИЕ}

Анализ динамики поступлений на лечение пациентов с дорсопатиями указал на то, что их количество за последние пять лет увеличилось в 1,9 раза (табл. 1).

Наиболее часто дорсопатии встречались у трудоспособной категории населения (от 30 до 60 лет - 62,2\%), что имеет не только медицинское, но и социально-экономическое значение (табл. 2). 
Динамика поступлений пациентов с дорсопатиями (n=341)

\begin{tabular}{|c|c|c|c|c|c|}
\hline Год & 2009 & 2010 & 2011 & 2012 & 2013 \\
\hline$\%$ & 13,8 & 16,7 & $23,2 *$ & $24,0^{*}$ & $22,3 *$ \\
\hline
\end{tabular}

Также нами были проанализированы данные о наиболее часто вовлекаемых в патологический процесс отделах позвоночника. Среди выбранных 371 пациентов дорсопатии поясничного отдела позвоночника в виде основного диагноза выставлены в 341 случае, что составило 91,1\%, в
5,9\% - поражался шейный отдел, в 3,0\% - грудной отдел позвоночника.

При более детальном анализе установлено, что на повторном лечении находилось 40,0\% пациентов. Из них 71,4\% имели стаж заболевания более 5 лет (табл. 3).

Распределение пациентов с дорсопатиями по возрастным группам (n=341)

\begin{tabular}{|c|c|c|c|c|c|c|}
\hline Возраст, лет & $18-30$ & $31-40$ & $41-50$ & $51-60$ & $61-70$ & $>70$ \\
\hline Количество пациентов, \% & 6,5 & 16,0 & $23,5^{*}$ & 22,7 & 19,9 & 11,4 \\
\hline $\begin{array}{l}\text { Количество пациентов с обострениями } \\
\text { более } 2 \text { раз в год, \% }\end{array}$ & $\mathbf{0}$ & $\mathbf{0}$ & $35,0^{*}$ & $35,1^{*}$ & 12,0 & 17,9 \\
\hline
\end{tabular}

При мечание $. *-p<0,05$.

При этом отмечался значительный рост обострений после 10-летнего стажа заболевания, который наиболее часто приходился на возраст 4160 лет $(70,1 \%)$.

Наличие более двух обострений в год обуславливает до двух месяцев нетрудоспособности, что является социально значимым фактором, учитывая распределение данной категории пациентов на наиболее трудоспособный возраст (41-60 лет).

Наиболее частыми клиническими проявлениями при дорсопатиях были: болевой синдром, МТ синдром и синдром СДН. При поступлении жалобы на боль предъявляли 97,9\% пациентов. В процессе лечения отмечалась положительная динамика болевого синдрома (табл. 4).

Таблича 3

Распределение больных по стажу заболевания и повторности лечения (n=139)

\begin{tabular}{c|c|c|c|c}
\hline Стаж заболевания, лет & менее 3 & $3-5$ & $5-10$ & Более 10 \\
\hline Повторность лечения, $\%$ & 12,3 & 16,4 & 25,5 & $45,8^{*}$ \\
\hline При ме чан ие . ${ }^{*}-$ p $<0,05$.
\end{tabular}


Таблииа 4

Динамика болевого синдрома $(\mathrm{n}=341)$

\begin{tabular}{|c|c|c|c|c|}
\hline Болевой синдром & Нет & Слабый & Умеренный & Выраженный \\
\hline До лечения & $2,1 \%(6)$ & $44,5 \%(153)$ & $40,2 \%(137)$ & $13,2 \%(45)^{*}$ \\
\hline После лечения & $4,1 \%(11)$ & $59,9 \%(204)$ & $32,8 \%(112)$ & $4,1 \%(14)$ \\
\hline
\end{tabular}

Данные таблицы 4 указывают на незначительное уменьшение умеренного болевого синдрома после лечения (с 40,2\% до $32,8 \%$ ) при достоверном снижении выраженного болевого синдрома более чем в три раза (с $13,2 \%$ до $4,1 \%$, $\mathrm{p}<0,05)$, что может говорить о достаточно эффективном медикаментозном влиянии на воспалительный процесс.

СДН (нарушения объема движений) были выявлены до лечения у 90,9\% пациентов. В процессе лечения распределение пациентов по СДН представлены в таблице 5.

Распределение больных по степени нарушения объема движения в поясничном отделе позвоночника $(\mathrm{n}=310)$

\begin{tabular}{|c|c|c|c|}
\hline Нарушение объема движения & 1 степень & 2 степень & 3 степень \\
\hline До лечения & $7,9 \%$ & $45,7 \%$ & $37,3 \%$ \\
\hline После лечения & $24,2 \%$ & $46,6 \%$ & $20,1 \% *$ \\
\hline
\end{tabular}

Изменения объема движений в позвоночнике является одним из наиболее показательных критериев эффективности ЛФК. В процессе лечения у $66,7 \%$ больных сохранялись функциональные блоки позвоночно-двигательных сегментов II и III степени, что свидетельствует о малоэффективном влиянии стандартного лече- ния на СДН. Так, среди пациентов с I ст. СДН отсутствие динамики отмечалось в $41,4 \%$, со II ст. - в $45,5 \%$, с III ст. - в $43,0 \%$ случаев.

Анализ распределения больных в зависимости от анатомической локализации структурных изменений представлен в таблице 6.

Таблича 6

Распределение больных в зависимости от анатомической локализации структурных изменений межпозвонковых дисков $(\mathrm{n}=341)$

\begin{tabular}{l|c|c|c|c|c}
\hline \multicolumn{1}{c|}{ Локализация } & L1-L2 & L2-L3 & L3-L4 & L4-L5 & L5-S1 \\
\hline Грыжа & $1,8 \%$ & $2,3 \%$ & $6,2 \%$ & $24,0 \% *$ & $29,3 \% *$ \\
Протрузия & $7,3 \%$ & $16,4 \%$ & $44,9 \%$ & $63,9 \% *$ & $49,3 \% *$ \\
\hline
\end{tabular}

При иечание ${ }^{*}-\mathrm{p}<0,05$. 
Наиболее часто протрузии $(63,9 \%$ и $49,3 \%)$ и грыжи $(24,4 \%$ и $29,3 \%)$ межпозвоночных дисков встречались в позвоночно-двигательных сегментах L4-15, L5-S1 соответственно. Это сочетается с клиническими проявлениями в виде радикулопатий (L5 - 31,37\%, S1 - 37,2 \%) и люмбоишиальгий $(31,1 \%)$, что при составлении комплексов лечебной физкультуры может послужить основанием для акцентирования внимания на вектор декомпрессионной разгрузки данных сегментов. Как основной диагноз, радикулопатия выставлена у 226 пациентов (66,3\%). Из них множественные поражения (поражение двух и более корешков спиномозгового нерва) отмечались у 70 пациентов (20,5\%). По локализации преобладали поражения сегментов L5 и S1 (68,6\%).

Стенозирование спиномозгового и межпозвонкового канала во многом определяет выраженность клинических проявлений и играет большое значение при разработке тактики реабилитации с включением комплексов ЛФК. Наличие стенозирования спиномозгового канала отмечалось у 80 пациентов $(23,5 \%)$. Стенозирование межпозвонкового (фораминального) канала отмечалось у 87 пациентов $(25,5 \%)$.

Среди всех пациентов метод ЛФК назначили в $42,5 \%$ случаев. Ограничение применения ЛФК было обусловлено наличием сопутствующих заболеваний: гипертонической болезни в $31,0 \%$ случаев, остеоартроза - 20,0\%, сахарного диабета 2 типа $-9 \%$.

\section{ВЫВОДЫ}

Анализ структуры заболеваемости дорсопатиями поясничного отдела позвоночника среди пациентов трудоспособного возраста позволяет сделать следующие выводы:

1. Дорсопатии встречаются у наиболее трудоспособной категории населения, при этом поражается преимущественно поясничный отдел позвоночника.

2. Наличие более двух обострений в год обуславливает около двух месяцев нетрудоспособности, что является социально значимым фактором, учитывая распределение данной категории пациентов на наиболее трудоспособный возраст (41-60 лет).

3. Отмечается незначительное уменьшение умеренного болевого синдрома после лечения (с $40,2 \%$ до 32,8\%) при достоверном снижении выраженного болевого синдрома более, чем в три раза (с $13,2 \%$ до 4,1\%), что может говорить о достаточно эффективном медикаментозном влиянии на воспалительный процесс.

4. После лечения, которое включало применение общепринятых (стандартных) комплексов ЛФК, нарушение объема движения и наличие функциональных блоков 2 и 3 степени сохранялось у $60 \%$ пациентов. Это свидетельствует о малоэффективном влиянии на статико-динамические нарушения, проявления функциональных блоков позвоночно-двигательных сегментов, морфологические изменения стандартного лечения.

5. Наиболее часто протрузии $(63,9 \%$ и $49,3 \%)$ и грыжи $(24,0 \%$ и $29,3 \%)$ межпозвоночных дисков встречались в позвоночно-двигательных сегментах L4-15, L5-S1 соответственно. Это сочетается с клиническими проявлениями в виде радикулопатий (L5 - 31,4\%, S1 - 37,2\%) и люмбоишиальгий $(31,1 \%)$, что при составлении комплексов лечебной физкультуры может послужить основанием для акцентирования внимания на вектор декомпрессионной разгрузки данных сегментов.

6. Стенозирование спиномозгового и межпозвонкового канала во многом определяет выраженность клинических проявлений и играет большое значение при разработке тактики реабилитации с включением комплексов ЛФК. Наличие первичных стенозов за счет склерозирования или вторичных (функциональных, динамических, за счет патологии межпозвонкового диска) определяет долгосрочность и тактику лечения с позиции применения ЛФК. В ретроспективных данных нет необходимого количества статистически достоверной информации о локализации стеноза и его характере, что определяет перспективу дальнейших исследований.

7. Среди всех пациентов метод ЛФК назначили в $42,5 \%$ случаев. Ограничение применения ЛФК было обусловлено наличием сопутствующих заболеваний: гипертонической болезни в $31,0 \%$ случаев, остеоартроза - $20,0 \%$, сахарного диабета 2 типа $-9 \%$.

Перспективой дальнейших исследований является разработка на основании полученных данных дифференцированного алгоритма построения программ реабилитации с учетом локализации морфологических изменений, качественных и количественных признаков тяжести заболевания у больных с дорсопатиями поясничного отдела позвоночника. 


\section{СПИСОК ЛИТЕРАТУРЫ}

1. Епифанов В.А. Остеохондроз позвоночника (диагностика, лечение и профилактика): Руководство для врачей / В.А. Епифанов, А.В. Епифанов. - М.: Медпресс-информ, 2004. - 272 с.

2. Епифанов В.А. Остеохондроз позвоночника / В.А. Епифанов // Физкультура в профилактике, лечении и реабилитации. - 2005. - № 2 (11). C. 26-34.

3. Жук П.М. Остеохондроз позвоночника. Лечение и профилактика / П.М. Жук, И.Н. Стельмах, А.3. Нычик. - К. : Книга-плюс, 2003. - 140 с.

4. Жулев Н.М. Остеохондроз позвоночника: Руководство для врачей / Н.М. Жулев, Ю.Д. Бадзгарадзе, С.Н. Жулев. - СПб.: Изд-во Лань, 2001. - 592 с.

5. Мендель О.И. Дегенеративные заболевания позвоночника, их осложнения и лечение / О.И. Мендель, А.С. Никифоров // Рус. мед. журнал. - 2006. Т. 14, № 4. - С. 34-39.

6. Смирнов А.Ю. Поясничный стеноз. Обзор литературы и анализ собственных наблюдений / А.Ю. Смирнов, Д.Р. Штульман, Г.Ю. Евзиков // Неврол. журнал. - 1998. - № 4. - С. 27-31.

7. Справочник по формулированию клинического диагноза болезней нервной системы / под ред. В.П. Штока, О.С. Левина. - М.: МИА, 2006. - 520 с.
8. Халафян A.A. STATISTIKA 6. Статистический анализ данных / А.А. Халафян. - М.: ООО «БиномПресс», 2007. - 512 c.

9. Lumbar spinal stenosis: Conservative or surgical management? A prospective 10-year study / T. Amundsen, H. Weber, H. Nordal [et al.] // Spine. - 2000. Vol. 25. - P. 1424-1436.

10. Malmivaara A. Surgical or nonoperative treatment for lumbar spinal stenosis? A randomized controlled trial / A. Malmivaara, P. Slatis, M. Heliovaara // Spine. 2007. - Vol. 32. - P. 1-8.

11. Mense S. Pathophysiology of low back pain and transition to the chronic state - experimental data and new concepts / S. Mense // Schmerz. Der.-2001.-Vol. 15.-P. 413-420.

12. Tadokoro $\mathrm{K}$. The prognosis of conservative treatments for lumbar spinal stenosis: Analysis of patients over 70 years of age / K. Tadokoro, H. Miyamoto, T. Shimomura // Spine. - 2005. - Vol. 30. - P. 2458-2463.

13. Wasiak R. Work disability and costs caused by recurrence of low back pain: longer and more costly than in first episodes / R. Wasiak, J. Kim, G. Pransky // Spine. - 2006. - Vol. 31, N 2. - P. 219-225.

14. Woolf A.D. The bone and joint decade 2000-2010 / A.D. Woolf // Ann. Rheumatic Disease. - 2000. Vol. 59. - P. 81-82.

\section{REFERENCES}

1. Epifanov VA, Epifanov AV. [Osteochondrosis (diagnosis, treatment and prevention): A Guide for Physicians]. Moscow: «Medpress-inform». 2004;272. Russian.

2. Epifanov VA. [Osteochondrosis of spine]. Fizkul'tura $\mathrm{V}$ profilaktike, lechenii i reabilitatsii. 2005;2(11):26-34. Russian.

3. Zhuk PM, Stel'makh IN, Nychik AZ. [Osteochondrosis of spine. Treatment and prevention]. Kiev: «Kniga-plyus». 2003;140. Russian.

4. Zhulev NM, Badzgaradze YuD, Zhulev SN. [Osteochondrosis: A Guide for Physicians]. St. Petersburg: Izdatel'stvo Lan'. 2001;592. Russian.

5. Mendel' OI, Nikiforov AS. [Degenerative diseases of the spine, their complications and treatment]. Russkiy meditsinskiy zhurnal. 2006;14(4):34-39. Russian.

6. Smirnov AYu, Shtul'man DR, Evzikov G.Yu. [Lumbar stenosis. Literature review and analysis of our own observations]. Nevrologicheskiy zhurnal. 1998;4:2731. Russian.

7. Shtok VP, Levin OS, editors. [Reference to formulate a clinical diagnosis of the nervous system of diseases]. Moscow: MIA. 2006;520. Russian.
8. Khalafyan AA. [STATISTICA 6. Statistic analysis]. Moscow. «Binom-Press». 2007;512. Russian.

9. Amundsen T, Weber H, Nordal H. Lumbar spinal stenosis: Conservative or surgical management? A prospective 10-year study. Spine. 2000;25:1424-36.

10. Malmivaara A, Slatis P, Heliovaara M. Surgical or nonoperative treatment for lumbar spinal stenosis? A randomized controlled trial. Spine. 2007;32:1-8.

11. Mense S. Pathophysiology of low back pain and transition to the chronic state - experimental data and new concepts. Schmerz. Der. 2001;15:413-20.

12. Tadokoro K, Miyamoto H, Shimomura T. The prognosis of conservative treatments for lumbar spinal stenosis: Analysis of patients over 70 years of age. Spine. 2005;30:2458-63.

13. Wasiak R, Kim J, Pransky G. Work disability and costs caused by recurrence of low back pain: longer and more costly than in first episodes. Spine. 2006;31(2):219-25.

14. Woolf AD. The bone and joint decade 2000-2010. Annals of Rheumatic Disease. 2000;59:81-2.

Стаття надійшла до редакції 23.10 .2014 